\title{
Saúde Mental, trabalho e aposentadoria: focalizando a alienação mental
}

\author{
Mental Health, work and retirement: a focus on mental allienation \\ Salud Mental, trabajo y jubilación: un enfoque en la alienación mental
}

\author{
Francisco Arnoldo Nunes de Miranda', Gysella Rose Prado de Carvalho', \\ Rafaella Leite Fernandes', Marta Batista Silvall, Maria das Graças Garcia Sabino ${ }^{\text {III }}$ \\ 'Universidade Federal do Rio Grande do Norte. Programa de Pós-Graduação em Enfermagem. Natal, RN \\ "Ministério da Saúde. Brasília, DF \\ "I'Secretaria de Estado da Saúde Pública. Centro de Apoio Psicossocial. Natal, RN
}

Submissão: 04/05/2009

Aprovação: $31 / 08 / 2009$

\section{RESUMO}

Estudo descritivo-exploratório Que objetivou analisar a aposentadoria por invalidez dos servidores da UFRN no período de $2000-2005$ por transtornos mentais e comportamentais. A amostra foi obtida nos registros no DAS/PRH/UFRN. Das 43 aposentadorias, $58 \%$ eram homens; 41\% aposentaram-se entre 41-50 anos e 35\% entre 51-60 anos; os Quais, 44\% ocupavam cargo/função do nível médio e $28 \%$ do nível elementar. Os transtornos do humor causaram 61 \% das aposentadorias por alienação mental, seguido dos transtornos do pensamento (19\%), os transtornos mentais orgânicos e os de personalidade (4\%). Conclui-se Que conviveram com intenso sofrimento psíquico gerando um ônus indireto em sua vida e de seus familiares frente às oportunidades perdidas de vida e aos anos ajustados de incapacitação afetando o trabalho.

Descritores: Pesquisa em enfermagem; Saúde mental; Saúde do trabalhador; Transtorno mental.

\section{ABSTRACT}

It is an exploratory-descriptive study that aimed to analyze the disability retirement of employees of the UFRN for mental and behavioral disorders in the period 2000-2005. The sample was obtained from records of the DAS / PRH / UFRN. Of 43 retirees, $58 \%$ were men, $41 \%$ retire between $41-50$ years and 35\% between $51-60$ years, $44 \%$ occupied position / function for the high school level and $28 \%$ for the elementary level. The mood disorders lead to $61 \%$ of the retirements by mental alienation, followed by thought disorders (19\%), organic mental disorders and personality disorder (4\%). We found that they lived with intense psychological distress creating an indirect burden on their lives and on their family's life facing lost opportunities of life and to the adjusted years of disability affecting their work. Descriptors: Nursing research; Mental health; Occupational health; Mental disorders.

\section{RESUMEN}

Estudio exploratorio-descriptivo Que objetivó analizar las jubilaciones por incapacidad de los servidores de la UFRN en el periodo 2000-2005 por los trastornos mentales y del comportamiento. La muestra se obtuvo en los registros de la DAS / PRH / UFRN. De las 43 jubilaciones, 58\% eran hombres, 41\% tenía entre 41-50 años y 35\% entre 51-60 años, Que, 44\% ocupaba puesto/función del nivel medio y $28 \%$ del nivel elemental. Los trastornos del estado de ánimo causado el $61 \%$ de las jubilaciones por alienación mental, seguido por el trastorno del pensamiento (19\%), trastornos mentales orgánicos y de la personalidad (4\%). Se concluye Que vivían con el sufrimiento psíquico intenso generando una carga indirecta en sus vidas y la de sus familias frente a la pérdida de oportunidades y de años de vida ajustados por discapacidad Que afectan lo trabajo.

Descriptores: Investigación en enfermería; Salud mental; Salud laboral; Transtornos mentales. 


\section{INTRODUÇÃO}

A conjuntura atual do país circunscreve um cenário de intensa e contínua deterioração das condições de vida e trabalho decorrente das condições e da atividade de trabalho, das relações intersubjetivas e suas implicações na saúde mental dos trabalhadores. A Saúde Mental no Trabalho é um campo de estudo interdisciplinar, de importância singular para a análise das situações de trabalho e suas implicações no campo da saúde mentall ${ }^{(1)}$.

O termo saúde mental está intimamente ligado ao de normalidade. Normalidade e saúde mental são Questões centrais na teoria e na prática psieuiátrica, mas são difíceis de definir, pois as manifestações comportamentais estão inscrita num Quadro de referência cultural do Qual revela a forma como a pessoa é aceita pela e na sociedade. Na perspectiva psicanalítica, normalidade neste estudo, diz respeito à capacidade da pessoa de desenvolver sentimentos sociais e de ser produtiva, pois a possibilidade de trabalhar eleva a auto-estima e torna o indivíduo capaz de se $\operatorname{adaptar}^{(2)}$.

Destarte, não se conhece o custo real, para o país, da ocorrência de acidentes e das doenças relacionados ao trabalho ${ }^{(3)}$. Não menos diferente Quando pensado em termo dos transtornos mentais e comportamentais.

No Brasil, em torno de $31 \%$ a $50 \%$ da população tende a apresentar durante a vida pelo menos um episódio de algum transtorno mental, cerca de $20 \%$ a $40 \%$ da população necessita, por conta desses transtornos, de algum tipo de ajuda profissional, indicando a relevância social da problemática. Leva-se em conta o real ônus das manifestações psicopatológicas e seus custos em termos humanos, sociais e econômicos, além da discriminação e da insuficiência dos serviços indispensáveis ao tratamento configurando o panorama geral dos transtornos mentais no mundo ${ }^{(4,5)}$

Os transtornos mentais na perspectiva da Saúde do Trabalhador constituem objeto desse estudo a partir do desfecho final, ou seja, da aposentadoria dos servidores de uma Instituição do Ensino Superior.

Sabe-se que, de um lado, o processo de afastamento do servidor, temporário e/ou definitivo do trabalho, por QualQuer motivo, gera um impacto negativo na vida do trabalhador. Do outro, Quando esse afastamento é decorrente das manifestações psicopatológicas dos transtornos mentais e comportamentais, adensando o estigma, o preconceito e a estigmatização em suas vidas, além das limitações impostas nas atividades da vida diária, reduz suas potencialidades na esfera do trabalho e da convivência familiar e social. Para tanto se faz necessário garantir os suportes sociais, educacionais e de saúde necessários para o seu reequilíbrio, assim como, o tratamento e o acesso aos serviços de saúde na manutenção terapêutica e nas crises.

Para este servidor, reinserir-se no contexto social requer, dentre outros, reajustes cognitivos, afetivos, psicomotores, além da credibilidade frente à família e a comunidade, o adicional de pertencimento a uma universidade Que por sua natureza pensa os problemas da sociedade através do ensino, pesQuisa e extensão propondo soluções para a mesma. Portanto, Quem são eles?

O presente estudo objetivou analisar a aposentadoria por invalidez dos servidores da Universidade Federal do Rio Grande do Norte no período de 2000-2005 com diagnósticos de transtornos mentais e comportamentais.

\section{REVISÃO DA LITERATURA}

A preocupação com a relação entre saúde e o modo como está organizado socialmente o trabalho, isto é, os espaços físicos institucionais e culturais de intervenção dos sujeitos entre si, encontram-se diretamente relacionado com as concepções do trabalho ${ }^{(1)}$

Segundo estimativa da OMS, os transtornos mentais leves acometem 30\% dos trabalhadores ocupados, e os transtornos graves acometem cerca de $5 \%$ a $10 \%$. No Brasil, dados previdenciários, mostram Que os transtornos mentais, com destaque para o alcoolismo crônico, ocupam o terceiro lugar entre as causas dessas ocorrências $^{(5)}$.

Em nossa sociedade, o trabalho é mediador de integração social, seja pelo seu valor econômico (subsistência) seja pelo aspecto cultural (simbólico), tendo, assim, importância fundamental na constituição da subjetividade, no modo de vida e, portanto, na saúde física e mental das pessoas. Em decorrência do lugar de destaque que o trabalho ocupa na vida das pessoas, sendo forte a garantia de subsistência e de posição social. A falta de trabalho ou mesmo a ameaça de perda de emprego geram sofrimento psíeuico, pois ameaçam a subsistência e a vida material do trabalhador e da sua família ${ }^{(3,6)}$.

Sem pretender maiores discussões teóricas, diagnósticas e legais, mas situar a problemática, a aposentadoria, por invalidez também entendida como pós-carreira independente das outras classificações, ocorre Quando a pessoa torna-se total e definitivamente incapaz para o trabalho, seja por motivo de doença ou acidente. Normalmente, o trabalhador Que adoece ou é acidentado, recebe benefícios como o auxílio-doença. Caso não tenha condições de retornar ao trabalho, é aposentado por invalidez após sucessivas avaliações pela perícia médico-legal. Se declarados insubsistentes os motivos determinantes da aposentadoria por invalidez, por Junta Médica Oficial, o servidor deverá retornar à atividade conforme prescreve o Art. 25 da Lei no 8.112/1990(7.8).

Destarte, a aposentadoria marca a passagem do servidor da atividade para a inatividade, com proventos integrais ou proporcionais, por estar incapacitado para o exercício de QualQuer atividade laboral. Sobre este último tipo, freeüente ocorre solicitação revisional da aposentadoria. Em ambos os casos, a aposentadoria ocorre mediante laudo do serviço de avaliação e perícia da saúde.

A aposentadoria por invalidez decorrente de transtornos mentais caracteriza-se como todo caso de distúrbio mental ou neuromental grave e persistente no Qual, esgotados os meios habituais de tratamento (psicoterapia, psicofarmacoterapia, terapêutica biológica (eletroconvulsoterapia, insulinoterapia, etc), haja alteração completa ou considerável da personalidade, comprometendo gravemente os juízos de valor e realidade, destruindo a autodeterminação do pragmatismo e tornando o paciente total e permanentemente impossibilitado para Qualeuer trabalho ${ }^{(9)}$.

Dito num linguajar corrente entre as pessoas Que vivenciam esta condição significa Que o portador é "doido de pedra, mesmo e não há mais nada a fazer". Nesse sentido a III Conferência Nacional de Saúde Mental recomenda a adeeuação da terminologia adotada 
pela Previdência Social e o Código Civil à Lei N. ${ }^{\circ} 0.2$ I6/01, de modo a suprimir expressões como "loucos de todo o gênero", assegurando ao portador de transtorno mental uma legislação Que o reconheça como sujeito de direitos ${ }^{(10)}$.

Paradoxalmente, o termo alienação mental como situação de aposentadoria vai de encontro à terminologia recomendada pela Organização Mundial de Saúde - OMS, para não reforçar o sentido preconceituoso Que o termo encerra, mas latinizá-lo, sem menosprezar sua gravidade frente à incapacitação e das oportunidades perdidas de vida, mas, assegurando a inclusão social e a ampliação da cidadania aos portadores de transtornos mentais e comportamentais ${ }^{(5)}$.

Na concepção legal para o termo Alienação Mental, predominam os Quadros clínicos: o transtorno intelectual - atinge as funções mentais em conjunto e não apenas algumas delas; a falta de autoconsciência - o paciente ignora o caráter patológico de seu transtorno ou tem dele uma noção parcial ou descontínua; a inadaptabilidade - o transtorno mental é evidenciado pela desarmonia de conduta do paciente em relação às regras Que disciplinam a vida normal em sociedade; e, a ausência de utilidade - a perda da adaptabilidade redunda em prejuízo para o paciente e para a sociedade. Considera-se, ainda os estados demenciais (senil, pré-senil, arterioesclerótica, luética, coréica, doença de Alzheimer e outras formas bem definidas); as psicoses esquizofrênicas nos estados crônicos; a paranóia e a parafrenia nos estados crônicos; e as oligofrenias graves ${ }^{(7,8)}$.

Entende-se como Transtornos Mentais e Comportamentais as condições clinicamente significativas caracterizadas por alterações do modo de pensar e do humor (emoções) ou por comportamentos associados com angústia pessoal e/ou deterioração do funcionamento ${ }^{(5)}$.

\section{METODOLOGIA}

Estudo exploratório descritivo de abordagem euantitativa. A amostra sobre aposentadoria por invalidez por transtornos mentais dos servidores da UFRN foi obtida nos registros do Departamento de Assistência à Saúde (DAS), Pró-Reitoria de Recursos Humanos - PRH/UFRN, referente ao período de 2000 a 2005, totalizando 43 aposentadorias.

Trata-se de um estudo parcial de natureza descritiva com abordagem Quantitativa. Os dados foram coletados nas fichas e boletins areuivados no DAS/PRH/UFRN, agrupados e analisados a partir do CID 10, DSM-IV-TR" ${ }^{11}$. Com base no Capítulo V da CID10 dedicado exclusivamente aos Transtornos Mentais e Comportamentais, além de nominar as doenças e os distúrbios, como os demais capítulos, ele inclui também descrições clínicas e critérios de diagnóstico para pesQuisa $^{(5)}$.

\section{RESULTADOS}

Após coleta de dados realizada nas fichas e boletins e o agrupamento e análise das categorias foi realizada a análise e discussão dos mesmos. Sabe-se que os Transtornos Mentais podem causar incapacidade grave e definitiva, inclusive a incapacidade de trabalhar ${ }^{(5)}$.

Concede-se a aposentadoria por invalidez Quando for resultante da classificação por doença especificada no $\S 1^{\circ}$ do Art. 186, da Lei no 8.112/1990, Que diz respeito à doença profissional ou acidente em serviço, os proventos serão integrais, independente do tempo de serviço. A aposentadoria por invalidez vigorará a partir da data da publicação do respectivo ato, segundo o Art. 188 da Lei $n^{\circ} 8.112 / 1990^{(7)}$.

Quando a aposentadoria proporcional por invalidez não for motivada pelas doenças especificadas no $\S 1^{\circ}$ do Art. 186 da Lei $n^{\circ} 8.11$ 2/90, ou seja, tuberculose ativa, alienação mental, esclerose múltipla, neoplasia maligna, cegueira posterior ao ingresso no serviço público, hanseníase, cardiopatia grave, doença de Parkinson, paralisia irreversível e incapacitante, espondiloartrose ancuilosante, nefropatia grave, estados avançados do mal de Paget (osteiti deformante), Síndrome de Imunodeficiencia Adquirida - AIDS, e outras que a Lei indicar, com base na medicina especializada; os proventos serão proporcionais ao tempo de serviço, mas nunca inferior a I/3 (um terço) da remuneração da atividade. (Art. $186 \mathrm{e}$ 191 da Lei no 8.112/90).

Ressalta-se Que uma incidência de comportamento anormal ou um curto período de anormalidade do estado afetivo não significa, em si, a presença de distúrbio mental ou de comportamento. Para serem categorizadas como transtornos, é preciso Que essas anormalidades sejam sustentadas ou recorrentes e Que resultem em certa deterioração ou perturbação do funcionamento pessoal, em uma ou mais esferas da vida ${ }^{(5)}$.

Para este recorte os servidores aposentados por transtorno mental na UFRN foram 43 servidores, dos Quais $58 \%$ são do gênero masculino e $42 \%$ do feminino. Destes, $44 \%$ ocupavam cargo/função do nível médio, $28 \%$ do nível elementar, $21 \%$ do nível superior e $7 \%$ dos docentes. Os Transtornos Mentais estão presentes em mulheres e homens em todos os estágios da vida ${ }^{(5)}$.

Quanto à idade dos servidores aposentados da UFRN, 4I\% aposentaram-se com idade entre 41 a 50 anos, $35 \%$ na faixa de 51 a 60 anos. Somados correspondem a $78 \%$. Abaixo dos 40 anos somente $12 \%$ e igual percentual para aQueles acima dos 60 anos.

Os resultados apontam para um período de intensa incapacitação, sofrimento e muitas idas e vindas aos serviços de saúde mental e perícia médica até a emissão do laudo conclusivo sobre seu sofrimento psíquico. No Que diz respeito aos transtornos mentais e comportamentais determinantes para a aposentadoria por invalidez nos laudos devem constar, obrigatoriamente, o termo alienação mental, esclarecendo o grau ou intensidade da enfermidade e o estágio evolutivo da doença, exemplificando: Esquizofrenia Paranóide, F.20.0, estágio pré-terminal grave, (Alienação Mental).

Todos os seres humanos estão familiarizados com as emoções de alegria e tristeza. Os adultos saudáveis experimentam essas emoções de um modo previsível, geralmente em resposta a um estímulo externo. Em contraposição, os indivíduos Que sofrem de transtornos do humor experimentam um grau profundo de alegria ou tristeza aparentemente não relacionado com estímulos externos e por um longo período. Além disso, invadem todo o ser e podem flutuar amplamente de uma para outra ${ }^{(12)}$.

Os dados sobre os diagnósticos definidores da aposentadoria por invalidez decorrente de Alienação Mental são demonstrados e discutidos a partir das tabelas com dados numéricos brutos e suas 
percentagens, respeitado os diversos agrupamentos classificatórios dos transtornos mentais expressos no CID-10 DSM-III-R: transtornos do humor (depressão e mania) (Tabela I), transtornos do pensamento (Tabela 2), transtornos de personalidade (Tabela 3) e transtornos mentais orgânicos (Tabela 4). Por esta ordenação enfatiza-se a predominância dos mesmos no processo de aposentadoria dos servidores da UFRN.

Destaca-se a coexistência de mais de um diagnóstico no laudo da aposentadoria, pois não existem sintomas psicopatológicos totalmente específicos de um determinado transtorno, por não existir sintomas patognomônicos em psiquiatria ${ }^{(4)}$. A ocorrência simultânea de dois ou mais transtornos mentais no mesmo indivíduo é particularmente comum com o passar da idade, Quando diversos transtornos físicos e mentais podem ocorrer juntos, trata-se da comorbidade a Qual tem sérias repercussões na identificação, tratamento e reabilitação das pessoas afetadas ${ }^{(5)}$.

Neste sentido, os transtornos mentais e comportamentais são identificados e diagnosticados através dos métodos clínicos semelhantes aos utilizados para os transtornos físicos Que incluem uma cuidadosa anamnese colhida com o indivíduo e com outras pessoas, incluindo sua família; um exame clínico sistemático para definir o estado mental; e os testes e investigações especializadas Que se fizerem necessários ${ }^{(5)}$.

Observou-se Que os transtornos do humor foram responsáveis por $61 \%$ das aposentadorias por invalidez e alienação mental, seguido dos transtornos do pensamento com 19\%, os transtornos mentais orgânicos e, finalmente, com $4 \%$ os transtornos de personalidade, discutidos a seguir.

\section{Os Transtornos do Humor}

Os transtornos do humor são subdivididos em dois grupos diagnósticos clínicos como transtornos depressivos e manias, ambos, interferem seriamente na Qualidade de vida das pessoas e suas famílias por estarem ligados a afetividade. Além disso, chama-se atenção para a depressão Que aumenta o risco de morte por suicídio $^{(12)}$.

Majoritariamente, os Transtornos do Humor com $61 \%$ das aposentadorias, onde $40 \%$ são referentes aos estados depressivos e $21 \%$ aos maníacos foi à primeira causa de aposentadoria por invalidez por Alienação Mental, conforme a Tabela I. São de longe os transtornos de maior prevalência e a incidência frente aos demais diagnósticos. Chama-se a atenção para a co-morbidade no grupo das manifestações depressivas, tais como os transtornos mentais e comportamentais devidos ao uso de álcool [F I0] e a ciclotimia [F34].

A vida afetiva é a dimensão psíquica Que dá cor, brilho e calor a todas as vivências humanas ${ }^{(4)}$. O termo afetividade é genérico, compreende várias modalidades de vivências afetivas como o humor ou estado e ânimo, emoções, sentimentos, afetos e paixões. Vale destacar Que a depressão é essencialmente um transtorno episódico recorrente e a cada episódio geralmente dura de alguns meses a alguns anos, com um período normal interveniente, onde se estima Que 20\% dos casos seguem um curso crônico e sem remissão, especialmente Quando não há tratamento adequado disponível ${ }^{(5)}$.

\section{Os Transtornos do Pensamento}

A capacidade de um indivíduo para lidar subjetivamente e em termos comportamentais com as demandas da vida em uma sociedade complexa, globalizada e conflitante pressupõe Que ele seja capaz de filtrar, processar e adaptar-se a um número infinito de estímulos internos e externos com os Quais é continuadamente bombardeado ${ }^{(12)}$. O transtorno do pensamento conhecido atualmente como esQuizofrenia existiu indubitavelmente ao longo de toda a história humana.

Os Transtornos do Pensamento, ou seja, Esquizofrenia, transtornos esQuizotípicos e delirantes com 19\% foi a segunda causa de aposentadoria por invalidez por Alienação Mental (Tabela 2).

A esquizofrenia é uma das psicoses funcionais, cujos sintomas são classificados como positivos e negativos os Quais o indivíduo deve exibir sintomas característicos, no mínimo por seis meses. Os positivos refletem a presença de comportamento incomum, especificamente distorções no conteúdo e na forma de pensamento (delírios) e percepção (alucinações). Os negativos refletem a ausência do comportamento normalmente esperado, de maneira específica nas dimensões de afeto, definição de si mesmo, volição, relações interpessoais e, em alguns casos, comportamento psicomotor ${ }^{(12)}$.

Considera-se, ainda, Que a esQuizofrenia segue um curso variável, com completa recuperação sintomática e social em cerca de um terço dos casos, porém, pode seguir um curso crônico ou recorrente, com sintomas residuais e recuperação social incompleta. Mesmo Quando desaparecem os sintomas mais importantes, a pessoa demonstra falta de interesse e iniciativa no trabalho e nas atividades do cotidiano prejudicando sua competência social(5).

\section{Os Transtornos Mentais Orgânicos}

As síndromes demenciais, confusionais agudas (delirium) e psicogânicas focais ou sintomáticos formam um grupo de Quadros mentais e comportamentais Que, apesar de terem uma etiologia orgânica indiscutível e, muitas vezes, identificável, são estudadas pela psiquiatria e pela psicopatologia, devido ao fato de suas manifestações clínicas constituírem predominantemente sintomas mentais e comportamentais ${ }^{(4)}$.

Os Transtornos Mentais Orgânicos, inclusive os Sintomáticos com $11 \%$ foi a terceira causa de aposentadoria por invalidez por Alienação Mental (Tabela 3).

O Quadro clínico dos sintomas mentais e comportamentais manifesta-se, basicamente, por rebaixamento do nível de consciência Que reflete na sensopercepção, no humor, na psicomotricidade e na orientação afetam o estado de alerta prejudicando a atenção traduzida nas dificuldades de concentração e de apercepção de si e do meio. Ressalte-se para este agrupamento, o delirium. De manifestações clínicas muito variadas de paciente para paciente e de um episódio para outro, constitui uma preocupação adicional(4, I3).

No delirium o paciente pode apresentar-se lúcido e orientado em um exame ou num dado recorte de tempo e poucos minutos depois apresentar-se sonolento, desorientado, muito ansioso, com ilusões ou alucinações visuais, geralmente no final da tarde e início da noite ${ }^{(4)}$.

\section{Os Transtornos de Personalidade}

A palavra personalidade é derivada do termo grego persona para descrever a máscara teatral utilizada por alguns atores dramáticos ${ }^{(13)}$. Progressivamente, tornou-se representante da pessoa atrás da máscara - a pessoa 'real', ou seja, "a totalidade de características de uma pessoa Quanto aos traços emocionais e 
Tabela 1. Servidores Aposentados da UFRN por Transtornos do Humor - CID 10-DSM-IIIR: 2000-2005.

\begin{tabular}{|c|c|c|c|c|c|}
\hline \multirow{2}{*}{ APOSENTADORIA POR ALIENAÇÃO MENTAL } & \multicolumn{3}{|c|}{ DIAGNÓSTICO } & \multirow{2}{*}{$\mathbf{N}$} & \multirow{2}{*}{$\%$} \\
\hline & CID-10 & CID-10 & CID-10 & & \\
\hline \multicolumn{6}{|l|}{ F30-F39 Transtornos do Humor [afetivos] - depressivos } \\
\hline Episodio depressivo moderado - transtornos mentais & F32.1 & Fl0.1 & - & 1 & 2 \\
\hline Episodio depressivo grave sem sintomas psicóticos & F32.2: & - & - & 2 & 5 \\
\hline Episódio depressivo grave com sintomas psicóticos. & F32.3 & - & - & 1 & 2 \\
\hline Transtorno depressivo recorrente sem sintoma psicótico & F33.3 & - & - & 9 & 21 \\
\hline Transtorno depressivo recorrente com episodio atual grave & F 33.2 & - & - & 1 & 2 \\
\hline Transtorno depressivo recorrente sem especificação & F33.9: & $\mathrm{F} 10.2$ & - & 1 & 2 \\
\hline Transtorno depressivo recorrente sem especificação & F 33.9 & - & - & 1 & 2 \\
\hline Transtorno depressivo recorrente sem sintoma psicótico & F33.3 & F34.8 & - & 1 & 2 \\
\hline \multicolumn{6}{|l|}{ Transtornos do Humor - [afetivos] manias } \\
\hline $\begin{array}{l}\text { Transtorno afetivo bipolar, episodio atual maníaco com } \\
\text { sintomas psicóticos }\end{array}$ & F31.2 & - & - & 1 & 2 \\
\hline $\begin{array}{l}\text { Transtorno afetivo bipolar, episodio atual depressivo com } \\
\text { sintomas psicóticos }\end{array}$ & $\mathrm{F} 31.5$ & - & - & 6 & 14 \\
\hline Outros transtornos afetivos bipolares & F 31.8: & - & - & 2 & 5 \\
\hline Outros episódios maníacos & $\mathrm{F} 30.8$ & - & - & 1 & 2 \\
\hline
\end{tabular}

Tabela 2. Servidores Aposentados da UFRN por Transtornos do Pensamento - CID I0-DSM-IIIR: 2000-2005.

\begin{tabular}{|c|c|c|c|c|c|}
\hline \multirow{2}{*}{ APOSENTADORIA POR ALIENAÇÃO MENTAL } & \multicolumn{3}{|c|}{ DIAGNÓSTICO } & \multirow{2}{*}{$\mathbf{N}$} & \multirow{2}{*}{$\%$} \\
\hline & CID-10 & CID-10 & CID-10 & & \\
\hline \multicolumn{6}{|c|}{ F20-F29 Transtornos do Pensamento - EsQuizofrenia, transtornos esQuizotípicos e delirantes } \\
\hline EsQuizofrenia transtorno esquizofrênico do tipo misto & $\mathrm{F} 25.2$ & F2l & - & 1 & 2 \\
\hline Psicose esquizofrênica e afetiva mista & F25.2 & - & - & 1 & 2 \\
\hline Esquizofrenia hebefrênica & F 20.1 & - & - & 1 & 2 \\
\hline EsQuizofrenia não especificada & F20.9 & - & - & 1 & 2 \\
\hline EsQuizofrênica paranóica & F20.0: & - & - & 3 & 7 \\
\hline $\begin{array}{l}\text { Transtorno psicótico agudo polimorfo, com sintoma } \\
\text { esQuizofrênicos }\end{array}$ & F23.I & FI0.5 & F34.0 & 1 & 2 \\
\hline Transtorno delirante & F22.0 & - & - & 1 & 2 \\
\hline
\end{tabular}

Tabela 3. Servidores Aposentados da UFRN por Transtornos Orgânicos, inclusive os sintomáticos - CID I0-DSM-IIIR: 20002005.

\begin{tabular}{|c|c|c|c|c|c|}
\hline \multirow{2}{*}{$\begin{array}{l}\text { APOSENTADORIA POR } \\
\text { ALIENAÇÃO MENTAL }\end{array}$} & \multicolumn{3}{|c|}{ DIAGNÓSTICO } & \multirow{2}{*}{$\mathrm{N}$} & \multirow{2}{*}{$\%$} \\
\hline & CID-10 & CID- 10 & CID-10 & & \\
\hline \multicolumn{6}{|l|}{ F00-F09 Transtornos Mentais Orgânicos, inclusive os Sintomáticos } \\
\hline Demência vascular mista & F01.3 & - & - & 1 & 2 \\
\hline $\begin{array}{l}\text { Demência em outras doenças especificadas classificadas em outra } \\
\text { parte }\end{array}$ & F02.8 & F06.6 & - & 1 & 2 \\
\hline Demência não especificada & $\mathrm{F} 03$ & - & - & 1 & 2 \\
\hline Doença de Alzheimer & F00. 1 & - & - & 2 & 5 \\
\hline
\end{tabular}

Tabela 4. Servidores Aposentados da UFRN por Transtornos de Personalidade - CIDI 0-DSM-IIIR: 2000-2005.

\begin{tabular}{|c|c|c|c|c|c|}
\hline \multirow{2}{*}{$\begin{array}{l}\text { APOSENTADORIA POR } \\
\text { ALIENAÇÃO MENTAL }\end{array}$} & \multicolumn{3}{|c|}{ DIAGNÓSTICO } & \multirow{2}{*}{$\mathbf{N}$} & \multirow{2}{*}{$\%$} \\
\hline & CIDIO & CIDI0 & CIDI0 & & \\
\hline F60-F69 Transtornos de Personalidade e do Comportamento do Adulto & & & & & \\
\hline $\begin{array}{l}\text { Transtornos mistos de personalidade e outros transtornos de } \\
\text { personalidade }\end{array}$ & F61 & - & - & 3 & 7 \\
\hline Transtornos de personalidade e irritabilidade emocional & F60.3 & F34.8 & - & 1 & 2 \\
\hline
\end{tabular}

comportamentais aparentes na vida cotidiana, uma totalidade Que é geralmente estável e previsível”(2).

O transtorno de personalidade foi nomeado de diferentes formas, entretanto o termo que se popularizou, indevidamente, entre os profissionais de saúde mental foi psicopatia. O transtorno, dito de outra forma, faz com Que o seu portador sofra e faça sofrer a sociedade e não aprende com a experiência, por apresentar uma marcante desarmonia Que se reflete no plano intrapsíquico como 
no plano das relações interpessoais ${ }^{(4)}$.

Os Transtornos de Personalidade e do Comportamento do Adulto manifesto com $9 \%$ foi à Quarta causa de aposentadoria por invalidez por Alienação Mental (Tabela 4). Observou-se o acréscimo do diagnóstico por ciclotimia.

Os transtornos de personalidade surgem na infância ou na adolescência e tendem a permanecer relativamente estável por toda a vida. As reações afetivas são claramente desarmônicas, tais como: afetividade, controle dos impulsos, o modo e estilo de relacionamento com os outros, dentre outros, contribuindo para um mau desempenho ocupacional e social. Podem ser do tipo paranóide, esquizóide, sociopatia, bordeline, histriônico, esQuizotípico, etc ${ }^{(2,4)}$

\section{CONCLUSÕES}

Os transtornos mentais representam Quatro das dez principais causas de incapacidade em todo o mundo e afetam 25\% da população em alguma fase da sua vida. Verifica-se Que a maior incidência de aposentadoria por transtorno mental está nos cargos de nível médio. Inferimos Que, independente da história pregressa, há no ambiente de trabalho um componente pouco aclarado, Que causa ou precipita as manifestações psicopatológicas Que se agrava no decorrer do tempo por não mais dispor de controle do seu processo de trabalho devido a ocupação funcional intermediária frente à hierarquização da IES.

Os transtornos mentais e comportamentais apresentam um Quadro variado e heterogêneo. Ao passo que alguns distúrbios são brandos, outros são graves. Alguns duram umas poucas semanas, outros podem durar a vida inteira. Alguns não chegam a ser seQuer percebidos, a não ser por um exame minucioso, ao passo Que outros são impossíveis de ocultar mesmo a um observador casual.

Contemporizando, infere-se que o servidor ao ser admitido na UFRN apresentava algum indício de transtorno do pensamento e de personalidade, com destaque, respectivamente para a eseuizofrenia e o comportamento anti-social. Do ponto de vista da ocorrência, no primeiro caso, inicia-se antes dos 25 anos, e a situação de crise gira em torno de 6 meses. No segundo, entre 25 e 44 anos.

E, ainda, levando-se em consideração a faixa etária Que vai dos 41 aos 50 anos como a maior taxa de aposentadoria por invalidez na UFRN, acrescente-se as sucessivas idas-e-vindas aos serviços de saúde e de perícia médica, há de se pensar que este servidor em plena fase produtiva do desenvolvimento conviveu com intenso sofrimento psíquico, cronificando-o, coisificando-o e o inacapacitando-o para o trabalho em si e para as atividades da vida diária afetando suas relações de parentalidade e afetividade, gerando um ônus indireto em sua vida e de seus familiares frente as oportunidades perdidas de vida frente aos anos ajustados de incapacitação.

\section{REFERÊNCIAS}

1. Ferreira SRV. Saúde mental e trabalho. João Pessoa: Editora Universitária; 2006

2. Sadock BJ, Sadock AS. Compêndio de psieuiatria - ciência do comportamento e psiQuiatria clínica. Porto Alegre: Artmed; 2007.

3. Dias EC, organizador. Doenças relacionadas ao trabalho: manual de procedimentos para os serviços de saúde. Brasília: Ministério da Saúde; 2001 .

4. Dalgalarrondo P. Psicopatologia e semiologia dos transtornos mentais. Porto Alegre: Artmed; 2000.

5. Organização Mundial da Saúde. Organização Panamericana da Saúde. Relatório sobre a saúde no mundo 2001: Saúde mental - nova concepção, nova esperança. Genebra: Organização Mundial da Saúde; 2001.

6. Mendes R. Patologia do trabalho. São Paulo: Atheneu; 1999.

7. Brasil. Regime Jurídico Único. Lei no 8. 1 12, de 11 de dezembro de 1990. Dispõe sobre o regime jurídico dos servidores públicos civis da União, das autarQuias e das fundações públicas federais. Diário Oficial da União 1990 dez 12.

8. Brasil. Presidência da República. Casa Civil. Lei n. 10.216, de 6 de abril de 2001. Dispõe sobre a proteção e os direitos das pessoas portadoras de transtornos mentais e redireciona o modelo assistencial em saúde mental. Brasília: Imprensa Oficial; 2003.

9. Ministério de Estado da Defesa (BR). Portaria Normativa No 328, de 17 de maio de 200 I. Brasília: Imprensa Oficial; 2001.

10. Ministério da Saúde (BR). Relatório Final da III Conferência Nacional de Saúde Mental. Brasília: Conselho Nacional de Saúde/Ministério da Saúde; 2002.

11. American Psychiatric Association. Diagnostic and Statistical Manual of Mental Disorders. $4^{\text {th }}$ ed. Washington: American Psychiatric; 2000.

12. Taylor CM. Fundamentos de enfermagem psiquiátrica de Mereness. Porto Alegre: Artes Médica; 1990.

13. Townsend MC. Enfermagem psiquiátrica - conceitos e cuidados. Rio de Janeiro: Guanabara-Koogan; 2000. 